\title{
Expresión de la ciclooxigenasa-2 en los carcinomas mamarios caninos primarios metastásicos y no metastásicos
}

\author{
Cyclooxigenase-2 expression in primary metastatic and non metastatic canine \\ mammarian carcinomas
}

\author{
AB De Nardi ${ }^{a^{*}}$, CR Daleck ${ }^{\mathrm{a}}$, RL Amorin ${ }^{\mathrm{b}}$, RR Huppes ${ }^{\mathrm{c}}$, RAR Uscategui ${ }^{\mathrm{d}}$, S Rodaski ${ }^{\mathrm{e}}$, C Calderon ${ }^{\mathrm{f}}$, RT Neto ${ }^{\mathrm{b}}$ \\ åDepartamento de Clínica y Cirugía Veterinaria, Universidad Estadual Paulista, Campus de Jaboticabal, São Paulo, Brasil. \\ bDepartamento de Clínica Veterinaria, Universidad Estadual Paulista, Campus de Botucatu, São Paulo, Brasil. \\ 'Doctorando del Programa de Clínica Veterinaria, Universidad Estadual Paulista, Campus Botucatu, São Paulo, Brasil. \\ ${ }^{d}$ Doctorando del Programa de Cirugía Veterinaria, Universidad Estadual Paulista, Campus Jaboticabal, São Paulo, Brasil. \\ eDepartamento de Medicina Veterinaria, Universidad Federal de Paraná, Campus Curitiba, Paraná, Brasil. \\ fUniversidad Estadual del Norte de Paraná, Bandeirantes, Paraná, Brasil.
}

\begin{abstract}
SUMMARY
The aim of this study was to detect the expression of ciclooxigenase-2 (COX-2) in metastatic primary carcinoma and non metastatic carcinoma, taking into consideration the relation between COX-2 and the progression of cancer. Evaluation of the COX-2 expression was achieved by immunohistochemistry analysis, using the primary polyclonal antibody anti-PGHS-2, clone PG 27, (Oxford Biomedical Research). The number of marked cells by the COX-2 antibody was higher $(\mathrm{P}<0.001)$ in the metastatic primary carcinoma $(81.25 \%)$ than non-metastatic $(60.3 \%)$. There was a positive correlation between the number of labeled cells.
\end{abstract}

Key words: cancer, mammary neoplasm, ciclooxigenase-2, dogs.

\section{RESUMEN}

Teniendo en cuenta la relación de la ciclooxigenasa-2 (COX-2) con la progresión del cáncer, el objetivo de este trabajo fue investigar la expresión de la COX-2 en los carcinomas mamarios primarios, metastásicos y no metastásicos en perras. La evaluación de la expresión de COX-2 fue realizada por inmunohistoquímica, utilizando anticuerpo policlonal primario anti-PGHS-2, clon PG 27, (Oxford Biomedical Research). El número de células marcadas por el anticuerpo COX-2 fue significativamente mayor $(\mathrm{P}<0.001)$ en los carcinomas primarios metastásicos $(81,25 \%)$ comparados con los no metastásicos $(60,3 \%)$. Existe correlación positiva entre el número de células marcadas COX-2 y el grado de invasión tumoral (carcinoma mamario primario metastásico y no metastásico).

Palabras clave: cáncer, neoplasia mamaria, ciclooxigenasa-2, perros.

\section{INTRODUCCIÓN}

El cáncer mamario es la neoplasia más común en perras (Zuccari 2001). Aproximadamente 50\% de estos tumores son malignos y el $25 \%$ de los casos presentan metástasis al momento del diagnóstico (Kitchell 1995, Morrison 1998). Generalmente las metástasis ocurren en los nódulos linfáticos, aunque pueden ocurrir también en otros órganos tales como hígado, corazón, riñón, piel, cerebro y hueso (Kitchell 1995).

Debido a la alta incidencia de metástasis al momento del diagnóstico en los pequeños animales, nuevos estu-

Aceptado: 20.03.2013.

* Vía de Acesso Prof. Paulo Donato Castellane, s/no, CEP 14884-900, Jaboticabal, San Paulo, Brasil; andrigobarboza@yahoo.com.br. dios vienen siendo realizados, con el objetivo de esclarecer los mecanismos ligados con la progresión neoplásica y las opciones terapéuticas que permitan bloquear estos mecanismos. Todo esto con el fin de mejorar el pronóstico de los animales acometidos por neoplasias mamarias (De Nardi y col 2009, De Nardi y col 2011).

Existe la posibilidad que la ciclooxigenasa 2 (COX-2) esté indirectamente relacionada con el desarrollo del cáncer. Esta correlación surgió a partir de varios estudios que observaron asociación entre el uso crónico de antinflamatorios no esteroides (AINES) y la disminución en la incidencia de carcinoma de colon en humanos y carcinomas de células transicionales de la vejiga urinaria en perros (Thun y col 1993, Knapp y col 1992, De Nardi y col 2011).

Normalmente la COX-2 no se encuentra en las células sanas (Davies 1995, Pires y col 2010), pudiendo ser 
inducida por reacciones inflamatorias, promotores tumorales, oncogenes y factores del crecimiento (Pires y col 2010, Queiroga y col 2010, De Nardi y col 2011). De este modo la sobre expresión de la COX-2 en tumores ha sido vinculada con peores pronósticos (Lascelles 2007). Estudios en medicina veterinaria demostraron aumento en la expresión de COX-2 y de prostaglandina E (PGE2) en los tumores mamarios (Dias Pereira y col 2009, Lavalle y col 2009, Queiroga y col 2010).

La PGE2 es el principal producto de la COX-2 implicado en el desarrollo y progresión de los tumores. Las células tumorales producen factores estimuladores de colina que activan los monocitos y los macrófagos provocando la síntesis de PGE2, de esta forma inhiben la acción de las linfocinas, de las células T y B y la acción cito tóxica de las células "natural killer". Al mismo tiempo la PGE2 también inhibe los factores de necrosis tumoral (TNF) e induce la producción de interleucuina-10 (IL-10), que promueve inmunosupresión (Dannenberg y col 2001, Mohammed y col 2001, Wang y col 2006, De Nardi y col 2011).

Según Souza y col (2000) la sobre expresión de la COX-2 en las células tumorales de mama en humanos se asocia a aumento en la expresión de metaloproteinasas, que posibilitan el rompimiento de las barreras residuales, por su capacidad de lisar colágeno tipo I, II, III y IV (presente en la membrana basal). Este mecanismo genera células con mayor potencial metastásico y capacidad de invasión (Souza y col 2000, Surh y col 2001, Bol y col 2002).

La COX-2 también participa en el metabolismo del ácido araquidónico, sintetizando prostaglandinas que están relacionadas con la producción del factor de crecimiento endotelial (VEGF) y consecuentemente con la angiogénesis, imprescindible para la mayoría de los tumores sólidos por proveer los nutrientes, garantizar el crecimiento y la sobrevivencia de los mismos. Por otro lado la angiogénesis es crucial para el establecimiento de metástasis tumoral (Knottenbelt y col 2006, Lavalle y col 2009, De Nardi y col 2011).

El aumento en la expresión de la COX-2 en canes con cáncer de mama fue relacionado a menor tiempo de sobrevida (Lavalle y col 2009). Dias Pereira y col (2009) constataron que la mayoría de las lesiones metastásicas estudiadas exhibían sobre expresión de la COX-2, al igual que las neoplasias primarias, correlacionando este hallazgo con angiogénesis y progresión metastásica (De Nardi y col 2011).

Teniendo en cuenta la relación de la COX-2 con el desarrollo del cáncer, este trabajo tuvo como objetivo investigar la expresión de esta enzima en los carcinomas mamarios metastásicos y no metastásicos de hembras caninas.

\section{MATERIAL Y MÉTODOS}

Para la realización de este estudio fueron seleccionadas 20 muestras de tejido mamario con carcinoma en hembras caninas, atendidas en el Servicio de Oncología en el Hospital Veterinario de la Universidad Federal de
Paraná. Estas muestras fueron divididas de acuerdo con la clasificación histopatológica y los hallazgos clínicos de los pacientes, en dos grupos: 10 casos de carcinoma mamario primario no metastásico y 10 casos de carcinoma mamario metastásico.

La expresión de la cicloxigensa-2 fue determinada por inmunohistoquímica, realizada de acuerdo con el protocolo del Laboratorio de Inmunohistoquímica del Servicio de Patología Veterinaria de la Facultad de Medicina Veterinaria y Zootecnia (FMVZ) - Campus de Botucatu, SP, Brasil, usando la técnica estreptoavidina-biotina-peroxidasa.

Los bloques de parafina fueron cortados por medio de un micrótomo de $3 \mu \mathrm{m}$ y extendidos en láminas histológicas, preparadas con cola líquida a base de organosilano (A3648 - Sigma), transfiriéndolos a envases de vidrio vertical que posteriormente fueron almacenados en incubadoras entre 55 y $60^{\circ} \mathrm{C}$ por 24 horas. En seguida las láminas se sometieron al proceso de desparafinización e hidratación. Para realizar la recuperación antigénica las láminas se colocaron en solución tampón de Citrato 10 $\mathrm{mM}, \mathrm{pH}$ 6,0, en un horno de micro-ondas por 15 minutos (3 ciclos de 5 minutos a potencia máxima).

El bloqueo de la peroxidasa endógena se consiguió por inmersión de las láminas en una solución de agua oxigenada al $3 \%$ por 20 minutos. Al final las láminas fueron incubadas en cámara húmeda con el anticuerpo (antiPGHS 2 - C-terminus, clone PG 27, Oxford Biomedical Research, EUA) en la proporción de 1:500, por 18 horas ("over-night"), a una temperatura de $4{ }^{\circ} \mathrm{C}$.

Como sistema para amplificación fue utilizado el Envision Dual Link (Dako cód. K4065, Dinamarca) por una hora, a temperatura ambiente. Como control negativo, el anticuerpo primario fue substituido por inmunoglobulina de conejo (Dako cód. X0903, Dinamarca). La solución cromogénica de DAB líquido fue utilizada (Dako cód. K34466, Dinamarca), de acuerdo con las indicaciones del fabricante. Las láminas fueron sometidas a contra coloración con Hematoxilina de Mayer y montadas con resina sintética (Permount) y lamínulas.

El conteo de las células marcadas por la COX-2 en el estudio inmunohistoquímico, fue realizado con la ayuda de un equipo analizador de imágenes (KS 300, versión 3.0 - Zeiss, Alemania) constituido por un microscopio (Leica DMLD, Alemania) acoplado a una cámara digital (Sony - DXV15A, China). Se contó un total de 5 campos para cada caso analizado, obteniendo un mínimo de 250 células, entre positivas y negativas, valor posteriormente convertido a porcentaje para análisis. El conteo de las células imunorreactivas fue realizado independiente de la intensidad de marcación.

El análisis de los datos fue realizado usando el programa 'Statistical Analysis System' (SAS 1995, EUA), comprobando la independencia de los resultados (presencia o ausencia de la expresión para COX-2) mediante una tabla de contingencia 2X2 (Prueba de Chi cuadrado, post test de Fisher's $\mathrm{P}<0,05$ y coeficiente de contingencia $=0,37$ ). 


\section{RESULTADOS}

El número de células marcadas por el anticuerpo COX-2 (Cuadro 1) fue significativamente mayor $(\mathrm{P}<0,001)$ en los carcinomas primarios metastásicos $(81,25 \%)$ comparados con los no metastásicos $(60,3 \%)$.

En la evaluación inmunohistoquímica se observó una correlación positiva y significativa $(\mathrm{P}<0,001)$ entre la expresión de COX-2 y el grado de invasión tumoral (carcinoma mamario primario metastásico y no metastásico).

Un gran número de células con marcada expresión de la enzima COX-2, fue observado, en la mayor parte de los casos de carcinomas primarios no metastásicos (figura 1) y metastásicos (figura 2), siendo que la inmunorreactividad para la enzima estudiada fue enteramente confinada al citoplasma de las células neoplásicas.

En las preparaciones inmunohistoquímicas de los carcinomas primarios metastásicos se evidenció una fuerte marcación de células neoplásicas al interior de los vasos linfáticos (émbolo neoplásico) (figura 3). En una de estas preparaciones fue observado que al interior de los acinos, las células perdieron la adhesión (criterio de malignidad) y presentaron mayor marcación de la COX-2 (figura 4).

\section{DISCUSIÓN}

La realización de trabajos dentro de esta línea de investigación se justifica debido a la elevada incidencia de neoplasias mamarias en las hembras caninas (Gornman y col 1995, Morrison 1998, Perez-Alanza y col 1998). Adicionalmente, los tumores que afectan a perros y gatos son modelos apropiados para el estudio de la biología del cáncer y la evaluación de nuevos agentes terapéuticos para el tratamiento de estas neoplasias, debido a que los animales presentan tumores con comportamientos semejantes a los que afectan al hombre (MacEwen 1990, Zuccari y col 2004).

La clasificación de los tumores mamarios realizada de acuerdo con el sistema TNM (Tumor/ Nódulos linfáticos/ Metástasis) propuesto por la Organización Mundial de la Salud (Owen 1980) fue fundamental para la evaluación, en este ensayo, de la posible progresión tumoral para los principales lugares de ocurrencia de metástasis, como lo son nódulos linfáticos y pulmones (Kitchell 1995).

Los resultados encontrados evidencian la importancia del estudio de marcadores celulares en las neoplasias

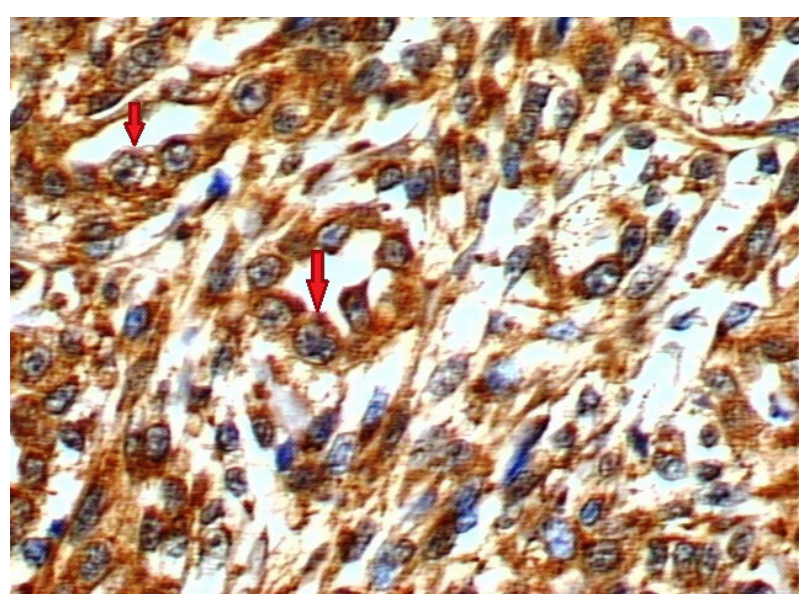

Figura 1. Fotomicrografía de preparación inmunohistoquímica que muestra un gran número de células neoplásicas marcadas por la actividad de la ciclooxigenasa-2 (flechas rojas) en un caso de carcinoma primario no metastásico de una hembra canina. 640X.

Photomicrographof immunohistochemical preparations showing a large number of neoplastic cells marked with cyclooxygenase-2 activity (red arrow) in a case of primary non metastatic carcinoma in a bitch. 640X.

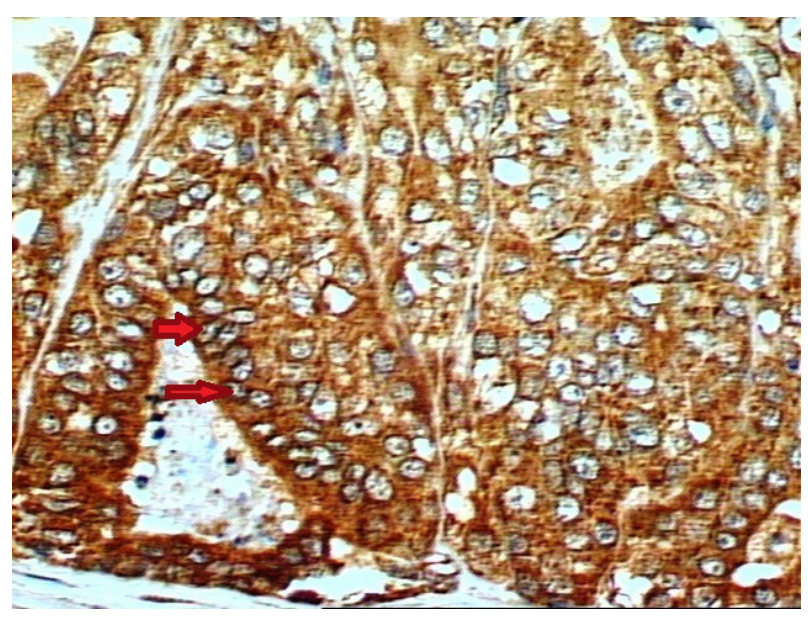

Figura 2. Fotomicrografía de preparación inmunohistoquímica que muestra un gran número de células neoplásicas marcadas por la actividad de la ciclooxigenasa-2 (flechas rojas) en un caso de carcinoma metastásico de una hembra canina. 640X.

Photomicrograph of immunohistochemical preparations demonstrating large number of neoplastic cells marked with cyclooxygenase- 2 activity (red arrow) in a case of metastatic carcinoma in a bitch. $640 \mathrm{X}$.

Cuadro 1. Resumen de las células marcadas con el anticuerpo COX-2 según el tipo de carcinoma y análisis estadístico. Summary of the antibody labeled cells with COX-2 by type of carcinoma and statistical analysis.

\begin{tabular}{|c|c|c|c|c|}
\hline Tipo de carcinoma & $\%$ células positivas COX-2 & $\%$ células negativas COX-2 & Valor de P & Indice de probabilidad \\
\hline No Metastásico & 60,30 & 39,70 & \multirow{2}{*}{$<0,001$} & \multirow{2}{*}{95,17} \\
\hline Metastásico & $81,25^{*}$ & 18,75 & & \\
\hline
\end{tabular}

*Representa diferencia significativa $(\mathrm{P}<0,05)$ entre los grupos.

"Represents significant difference $(\mathrm{P}<0.05)$ between groups. 


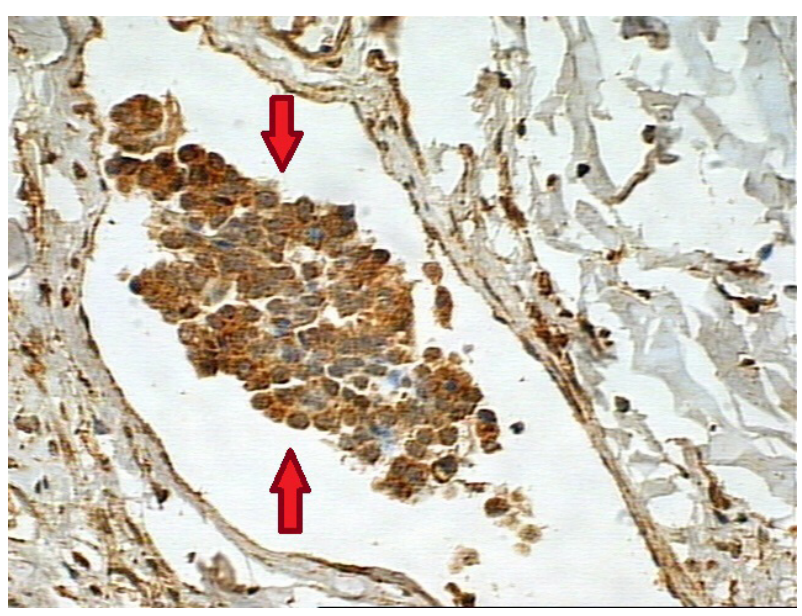

Figura 3. Fotomicrografía de preparación inmunohistoquímica que muestra fuerte marcación de las células neoplásicas en el interior de un vaso linfático (embolo neoplásico - flechas rojas), por la actividad de la ciclooxigenasa-2 en un caso de carcinoma metastásico en una hembra canina. 400X.

Photomicrograph of immunohistochemical preparations demonstrating stronglabeling of neoplastic cells within the lymphatic vessel (neoplastic plunger-red arrow) to the activity of cyclooxygenase- 2 in a metastatic carcinoma in a bitch. 400 times.

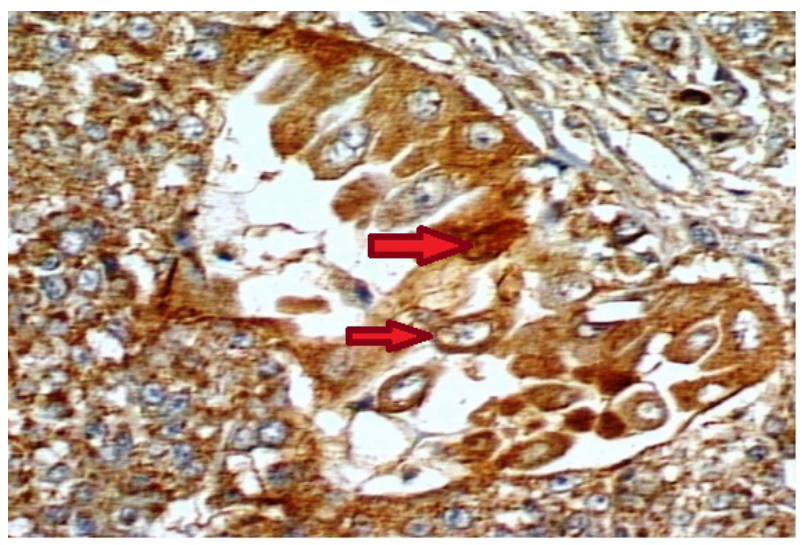

Figura 4. Fotomicrografía de preparación inmunohistoquímica que muestra células marcadas por la actividad de la ciclooxigenasa-2, con pérdida de adhesión al interior del acino (flechas rojas), en un caso de carcinoma metastásico en una hembra canina. 400X.

Photomicrograph of immunohistochemical preparations demonstrating cells labeling to the activity of cyclooxygenase- 2 with a loss of adhesion in the acino interior (red arrow), in a metastatic carcinoma in a bitch. 400X.

mamarias mediante la técnica de inmunohistoquímica (Zuccari 2001), pues fue posible determinar la presencia y expresión de la enzima COX-2; que normalmente es encontrada sólo como respuesta a estímulos inflamatorios (Souza y col 2000). La utilización de la técnica de la estreptoavidina - biotina peroxidasa, como recuperación antigénica en microondas, unida a la dilución del anti- cuerpo policlonal (PGHS-2 - C terminus, clon PG 27), en la proporción de 1:500 fue adecuada para realizar la inmunomarcación de las células neoplásicas.

El conteo de las células inmunorreactivas fue realizado de forma independientemente a la intensidad de inmunomarcación. La distinción entre células fuerte y débilmente positivas durante el conteo de las preparaciones inmunohistoquímicas puede ser subjetiva e inducir a errores en la evaluación (Scott 1991).

De acuerdo con ciertos trabajos (Bakhle 2001, Beam y col 2003) se puede especular que el desarrollo de la carcinogénesis mamaria está ligado a la actividad de la ciclooxigenasa-2, aunque la exacta implicancia de esta enzima en las neoplasias mamarias permanece desconocida. Algunos estudios epidemiológicos en caninos revelaron que el uso prolongado de antinflamatorios no esteroides, responsables de inhibir la COX-2, puede disminuir la incidencia de los carcinomas de células escamosas orales y de células transicionales de la vejiga urinaria (Beam y col 2003); sin embargo, esta acción aún no ha sido evaluada en canes con neoplasias mamarias.

Así como existe una correlación positiva entre la expresión de la COX-2 y la capacidad de invasión residual de las células tumorales, principalmente de colon y mama en humanos (Surh y col 2001, Bol y col 2002) se sugiere que puede existir una acción semejante con los carcinomas primarios metastásicos de las perras, debido al gran potencial de metástasis que estos tumores presentan.

Las metástasis pulmonares pueden haber sido facilitadas por la habilidad de la COX-2 para regular la angiogénesis, favoreciendo el aporte sanguíneo necesario para la diseminación de las células neoplásicas (Bakhle 2001, Tsujii y col 2003).

Futuras investigaciones deben ser realizadas con el objetivo de evaluar la asociación, entre la expresión de COX-2 y la presencia de receptores de estrógenos y progesterona en el tejido mamario. Siendo importantes factores para el pronóstico del cáncer de mama en caninos, puesto que las hormonas implicadas influyen en la carcinogénesis mamaria (Sartin 1992, MacEwen y col 1998, Morrison 1998, Fonseca 1999, Zuccari 1999).

Debido a la baja efectividad de los protocolos terapéuticos con fármacos antineoplásicos y radioterapia (Rutteman y col 2001), los tratamientos con inhibidores selectivos COX-2 pueden ser una alternativa importante para el tratamiento de las neoplasias mamarias en estos animales.

\section{REFERENCIAS}

Arber N. 2008. Cyclooxygenase-2 inhibitors in colorectal cancer prevention: point. Cancer Epidem Biomark 17, 1852-185.

Bakhle YS. 2001. Cox-2 and cancer: a new approach to an old problem. Br J Pharmacol 134, 1137-1150.

Beam SL, KM Rassnick, AS Moore, SP McDonough. 2003. An immunohistochemical study of ciclooxigenase-2 expression in various feline neoplasms. Vet Pathol 40, 496-500.

Bol DK, RB Rowley, CP Ho, B Pilz, J Dell, M Swerdel, K Ki- 
guchi, S Muga, R Klein, SM Fischer. 2002. Cyclooxygenase 2 over expression in the skin of transgenic mice results in suppression of tumor development. Cancer Res 62, 2516-2521.

Cao Y, SM Prescott. 2002. Many actions of cyclooxigenase-2 in cellular dynamics and in cancer. J Cell Physiol 190, 279-286.

Dannenberg AJ, NK Altorki, JO Boyle, C Dang, LR Howe, BB Weksler. 2001. Cyclooxygenase-2: a pharmacological target for the prevention of cancer. Lancet Oncol 2, 544-551.

Davies NM. 1995. Toxicity of nonsteroidal anti-inflammatory drugs in the large intestine. Dis Colon Rectum 38, 12, 1311-1321.

De Nardi AB, S Rodaski, NS Rocha, SC Fernandes. 2009. Neoplasias Mamárias. En: Dalcek CR, De Nardi AB, Rodaski S (eds). Oncologia em Cães e Gatos. Roca, São Paulo, Brasil, Pp 371-384.

De Nardi AB, TMM Raposo, RR Huppes, CB Daleck, RL Amorim. 2011. COX-2 Inhibitores for cancer treatment in dogs. Pak Vet J 31, 275-279.

Dias Pereira P, CC Lopes, AJ Matos, M Santos, F Gartner, R Medeiros, C Lopes. 2009. COX-2 expression in canine normal and neoplastic mammary gland. J Comp Pathol 140, 247-253.

Fonseca CS.1999. Avaliação dos níveis séricos de 17, $\beta$-estradiol e progesterona em cadelas portadoras de neoplasias mamárias. Disertación de Maestría, Faculdade de Ciências Agrárias e Veterinárias, Universidade Estadual Paulista, Jaboticabal, São Paulo, Brasil.

Kitchell BE. 1995. Mammary tumors. In: Bonagura JD, Abbott JA, Abrams KL (eds). Kirk's Current Veterinary Therapy XII Small Animal Practice. Philadelphia, USA, Pp 1098-1103.

Knapp DW, RC Richardson, GD Bottoms, R Teclaw, TCK Chan. 1992. Phase I trial of piroxicam in 62 dogs bearing naturally occurring tumors. Cancer Chemother Pharmacol 29, 214-218.

Knottenbelt C, D Mellor, C Nixon, H Thompson, DJ Argyle. 2006. Cohort study of COX-1 and COX-2 expression in canine rectal and bladder tumors. J Small Anim Pract 47, 196-200.

Lascelles BDX. 2007. Supportive care for the cancer patient. In: Withrow SJ, MacEwen EG (eds). Small Animal Clinical Oncology. $4^{\text {th }}$ ed. WB Saunders, St. Louis, MO, USA, Pp 291-306.

Lavalle GE, AC Bertagnolli, WLF Tavares, GD Cassali. 2009. Cox-2 Expression in canine mammary carcinomas: correlation with angiogenesis and overall survival. Vet Pathol $46,1275-1280$.

MacEwen EG, AK Patnaik, HJ Harvey, WB Panko.1982. Estrogen receptors in canine mammary tumors. Cancer Res, 42, 2255-2259.

MacEwen EG. 1990. Spontaneous tumors in dogs and cats: Models for the study of cancer biology and treatment. Cancer Metastasis Rev 9, 125-136.

Mohammed SI, K Coffman, NW Glickman, MG Hayek, DJ Waters, D Schlittler, DB DeNicola, D Knapp. 2001. Prostaglandin $\mathrm{E}_{2}$ concentretions in naturally occurring canine cancer. Prostaglandins, Leukot. Essent Fatty Acids 64, 1-4.

Morrison WB. 1998. Canine and feline mammary tumors. In: Morrison WB (ed). Cancer in Dogs and Cats. Williams and Wilkins, Baltimore, USA, Pp 591-598.
Perez Alenza D, GR Rutteman, L Peña, AC Beynen, P Cuesta. 1998. Relation between habitual diet and canine mammary tumors in a case-control stud. J Vet Inter Med 12, 132-139.

Pires I, A Garcia, J Prada, FL Queiroga. 2010. COX-1 and COX-2 Expression in canine cutaneous, oral and ocular melanocytic tumours. J Comp Path 143, 142-149.

Queiroga FL, MD Perez-alenza, G Silvan, L Pena, C Lopes, JC Illera. 2005. Cox-2 levels in canine mammary tumors, including inflammatory mammary carcinoma: clinicopathological features and prognostic significance. Antican Res 25, 4269-4275.

Queiroga FL, I Pires, L Lobo, CS Lopes. 2010. The role of Cox-2 expression in the prognosis of dogs with malignant mammary tumours. Res Vet Sci 88, 441-445.

Rossmeisl JH Jr, JL Robertson, KL Zimmerman, MA Higgins, DA Geiger. 2009. Cyclooxygenase 2 (COX-2) expression in canine intracranial meningiomas. Vet Comp Oncol 7, 173-180.

Rutteman GR, SJ Withrow, EG MacEwen. 2001. Tumors of the mammary gland. In: Withrow SJ, MacEwen EG (eds). Small Animal Clinical Oncology. $3^{\text {rd }}$ (ed). WB Saunders Company, Philadelphia, USA, Pp 455-477.

Sartin EA, S Barnes, RP Kwapien, LG Wolfe. 1992. Estrogen and progesterone receptor status of mammary carcinomas and correlation with clinical outcome in dogs. Am J Vet Res 53, 2196-2200.

Scott RJ, PA Hall, JS Haldane, S Noorden Van, Y Price, DP Lane, Na Wright. 1991. A comparison of immunohistochemical markers of cell proliferation with experimentally determined growth fraction. J Pathol 165, 173-178.

Souza CHM, AL Esteves, CA Cupello. 2000. Nonsteroidal Anti-Inflammatory Drugs (NSAIDs) and Cancer: Chemoprevention and Treatment. Vet Cancer Soc Newsl 10, 8-11.

Souza CHM, E Toledo-Piza, RL Amorin, AB De Nardi, KM Tobias. 2009. Inflammatory mammary carcinoma in 12 dogs: Clinical features, cyclooxygenase-2 expression, and response to piroxicam treatment. Can Vet J 50, 506-510.

Statistical Analysis System, SAS. 1995. SAS Institute Inc. Cary, NC, USA.

Surh YJ, KS Chun, HH Cha, SS Han, SY Keum, KK Park KK, SS Lee. 2001. Molecular mechanisms underlying chemopreventive activities of anti-inflammatory down-regulation of COX-2 and inos through suppression of NF-kB activation. Mutat Res 480-481, 243-268.

Thun MJ, SJ Henley, C Patrono. 1993. Nonsteroidal antiinflammatory drugs as anticancer agents: mechanistic, pharmacologic and clinical issues. J Nat Cancer Instit 94, 252-266.

Tsujii M, S Kawano, S Tsuji, H Sawaoka, M Hori, RN Dubois. 1998. Cyclooxygenase regulates angiogenesis induced by colon cancer cells. Cell 24, 705-716.

Wang LS, YW Huang, S Liu, HL Chang, W Ye, S Shu, Y Sugimoto, JA Funk, DD Smeaks, LN Hill, YC Lin. 2006. Conjugated linoleic acid (CLA) modulates prostaglandin E2 (PGE2) signaling in canine mammary cells. Antic Res 26, 889-898.

Zuccari DAPC. 1999. Contribuição ao estudo imunoistoquímico dos tumores mamários em cadelas Disertación de Maestría, Faculdade de Ciências Agrárias e Veterinárias, Universidade Estadual Paulista, Jaboticabal, São Paulo, Brasil. 
Zuccari DAPC. 2001. Estudo imunocitoquímico de marcadores diagnósticos e prognósticos em neoplasias mamárias caninas. Tesis Doctoral, Faculdade de Ciências Agrárias e Veterinárias, Universidade Estadual Paulista, Jaboticabal,
São Paulo, Brasil.

Zuccari DAPC, AE Santana, PM Cury, JA Cordeiro. 2004. Immunocytochemical study of $\mathrm{Ki}-67$ as a prognostic marker in canine mammary neoplasia. Vet Clin Pathol 33, 23-28. 